\title{
THE ENTROPY OF CHEBYSHEV POLYNOMIALS
}

\author{
BY \\ R. L. ADLER AND M. H. MCANDREW
}

1. Introduction. The purpose of this work is to compute the topological entropy of the $v$ th Chebyshev polynomial $T_{v}(x)$ considered as a map of $[-1,1]$ onto itself. The notation and basic definitions relevant to the concept of topological entropy are contained in [1] and are reviewed briefly below.

For an open cover $\mathfrak{A}$ of a compact space $X, N(\mathfrak{A})$ denotes the minimum cardinality of all sub-covers of $\mathfrak{A} . H(\mathfrak{U})=\log N(\mathfrak{U})$ is called the entropy of $\mathfrak{A}$. A cover $\mathfrak{B}$ is said to refine a cover $\mathfrak{A}$ if every set of $\mathfrak{B}$ is a subset of some set of $\mathfrak{A}$; we use the notation $\mathfrak{U} \prec \mathfrak{B}$. We define the join of two covers $\mathfrak{A}, \mathfrak{B}$ to be the cover $\mathfrak{A} \vee \mathfrak{B}=\{A \cap B ; A \in \mathfrak{U}, B \in \mathfrak{B}\}$. For a continuous map $\phi$ of $X$ into itself we define $h(\phi, \mathfrak{A})$, the entropy of $\phi$ with respect to $\mathfrak{A}$ to be

$$
\lim _{n \rightarrow \infty} H\left(\mathfrak{A} \vee \phi^{-1} \mathfrak{A} \vee \cdots \vee \phi^{-n+1} \mathfrak{A}\right) / n
$$

in [1] this limit is shown to exist. Finally $h(\phi)$, the entropy of $\phi$, is defined to be $\sup h(\phi, \mathfrak{A})$ where the supremum is taken over all open covers $\mathfrak{A}$ of $X$. In the sequel we use the following properties.

(1) $\prec$ is transitive.

(2) $\mathfrak{A} \prec \mathfrak{U}^{\prime}$ and $\mathfrak{B} \prec \mathfrak{B}^{\prime} \Rightarrow \mathfrak{A} \vee \mathfrak{B} \prec \mathfrak{U}^{\prime} \vee \mathfrak{B}^{\prime}$.

(3) $\mathfrak{A} \prec \mathfrak{B} \Rightarrow N(\mathfrak{U}) \leqq N(\mathfrak{B})$.

(4) $\mathfrak{A} \prec \mathfrak{B} \Rightarrow \phi^{-1} \mathfrak{U} \prec \phi^{-1} \mathfrak{B}$.

(5) $\phi^{-1}(\mathfrak{U} \vee \mathfrak{B})=\phi^{-1} \mathfrak{U} \vee \phi^{-1} \mathfrak{B}$

(6) Let $\mathfrak{A}_{n}$ be a refining sequence; i.e. a sequence of open covers such that $\mathfrak{A}_{n} \prec \mathfrak{U}_{n+1}$ and for every open cover $\mathfrak{B}$ there is some $\mathfrak{A}_{n}$ with $\mathfrak{B} \prec \mathfrak{A}_{n}$. Then $h(\phi)=\lim _{n \rightarrow \infty} h\left(\phi, \mathfrak{A}_{n}\right)$. These properties are proved in [1].

\section{Preliminary lemmas.}

LEMMA 1. Let $X$ be a compact topological space and $\mu$ a Borel measure on $X$. For an open cover $\mathfrak{B}$ of $X$, let $g(\mathfrak{B}, x)=1 / \sup \mu(B)$, the supremum being taken over all $B$ with $x \in B$ and $B \in \mathfrak{B}$. Then $\int_{X} g(\mathfrak{B}, x) d \mu \leqq N(\mathfrak{B})$.

Proof. $g(\mathfrak{B}, x)$ is measurable since $\{x: g(\mathfrak{B}, x)<\lambda\}=\bigcup_{\mu\left(B_{i}\right)>1 / \lambda} B_{i}$, an open set.

Let $\mathfrak{B}^{\prime}=\left\{B_{1}, B_{2}, \cdots, B_{N(\mathfrak{B})}\right\}$ be a subcover of minimal cardinality. For $x \in X$ let $B(x)$ be that $B_{i}$ of least index such that $x \in B_{i}$. Then $\left\{x: B(x)=B_{i}\right\}$ is just

Received by the editors March 15, 1965 
$B_{i} \cap \bar{B}_{1} \cap \bar{B}_{2} \cap \cdots \cap \bar{B}_{i-1}$ and is measurable. If $\mu\left(B_{i}\right)=0$ then $\mu\left\{x: B(x)=B_{i}\right\}=0$ and

$$
\int_{\left\{x: B(x)=B_{i}\right\}} g(\mathfrak{B}, x) d \mu=0 .
$$

If $\mu\left(B_{i}\right) \neq 0$ then

$$
\int_{\left\{x: B(x)=B_{1}\right\}} g(\mathfrak{B}, x) d \mu \leqq \int_{B_{i}} \frac{1}{\mu\left(B_{i}\right)} d \mu=1 .
$$

Since $X=\bigcup_{i=1}^{N(3)}\left\{x: B(x)=B_{i}\right\}$ the result of the lemma now follows.

Lemma 2. Let $v \geqq 2$. Then there is a function $\lambda(r)$ defined for integral $r \geqq 2$ with the following properties:

(i) $\lim _{r \rightarrow \infty} \lambda(r)=v$.

(ii) If $r \geqq 2$ and $\left\{I_{n} ; n \geqq 0\right\}$ is a sequence of real numbers satisfying

$$
I_{n+1}>v I_{n}-(v-1) I_{n-s+1}
$$

for $1 \leqq s \leqq r$ and $s \leqq n+1$, then

$$
\liminf _{n \rightarrow \infty} I_{n}^{1 / n} \geqq \lambda(r) .
$$

Proof. We shall show that the unique positive zero of

$$
f_{r}(x)=x^{r-1}-(v-1)\left(x^{r-2}+x^{r-3}+\cdots+1\right)
$$

has the properties required for $\lambda(r)$. We note that for $r>2, \lambda(r)$ is the positive zero other than 1 of $g_{r}(x)=(x-1) f_{r}(x)=x^{r}-v x^{r-1}+v-1$. Now $g_{r}(v)=v-1>0$, and $g_{r}\left(v-v^{2-r}\right)=v-1-v\left(1-v^{1-r}\right)^{r-1}$. Clearly $g_{r}\left(v-v^{2-r}\right) \rightarrow-1$ as $r \rightarrow \infty$. Hence for $r$ sufficiently large, $v-v^{2-r}<\lambda(r)<v$. This verifies (2.1).

To verify the second property of $\lambda(r)$ let $r \geqq 2$ and let $I_{n}$ be a sequence satisfying (2.2). Let $J_{n}=I_{n+1}-I_{n}(n \geqq 0)$. Then from (2.2) with $s=1, J_{n}>0$. Further

$$
J_{n}>(v-1)\left(J_{n-1}+J_{n-2}+\cdots+J_{n-s+1}\right)
$$

for $2 \leqq s \leqq r$ and $s \leqq n+1$. We shall show that for $n \geqq 0$,

$$
J_{n} \geqq J_{0} \lambda(r)^{n-r} \text {. }
$$

Since $f_{r}(1)=1-(v-1)(r-1) \leqq 0$ and $f_{r}(+\infty)=+\infty, \lambda(r) \geqq 1$. Hence (2.6) is true for $n=0$. From (2.5) with $s=2,3, \cdots, r-1$ and $n=s-1$ it follows that $J_{n}>J_{0}$ for $1 \leqq n \leqq r-2$ and, a fortiori, (2.6) is true. The remaining cases for $n$ follow from (2.5) with $s=r$ by induction, since $\lambda(r)$ is a zero of (2.4). Relation (2.3) now follows from (2.6) since $I_{n}=I_{1}+\sum_{m=1}^{n-1} J_{m}$. 
3. Main result. Let $X$ be the interval $[-1,1]$ with its usual topology and let $\phi$ be the map $x \rightarrow T_{v}(x)$ where $T_{v}$ is the $v$ th Chebyshev polynomial; i.e. $T_{v}(\cos \theta)$ $=\cos v \theta$.

\section{THEOREM. $h(\phi)=\log v$.}

Proof. Let $X^{\prime}=[0, \pi]$ and let $\sigma$ be the homeomorphism of $X$ onto $X^{\prime}$ defined by $x^{\prime}=\sigma x=\cos ^{-1} x$. Let $\psi$ be the continuous map $\sigma \phi \sigma^{-1}$ of $X^{\prime}$ onto $X^{\prime}$. By Theorem 1 of [1], $h(\psi)=h(\phi)$ and so we may work with $\psi$ instead of $\phi$. The map $\psi$ is given explicitly by $\psi\left(x^{\prime}\right)=S_{v}\left(x^{\prime}\right)$ where

$$
S_{v}(x)=\left\{\begin{array}{l}
v x-k \pi, \quad k \text { even, } \\
(k+1) \pi-v x, \quad k \text { odd },
\end{array}\right.
$$

for $k \pi / v \leqq x \leqq(k+1) \pi / v, k=0,1, \cdots, v-1$. Figure 1 illustrates the case $v=3$. Now $S_{1}$ is just the identity transformation on $X^{\prime}$ and hence for $v=1, h(\psi)=0$.

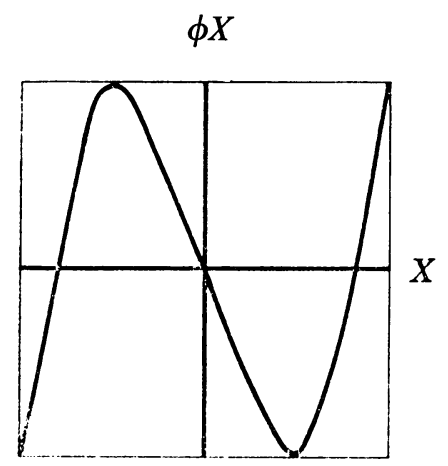

$\phi X^{\prime}$

\section{FIGURE 1}

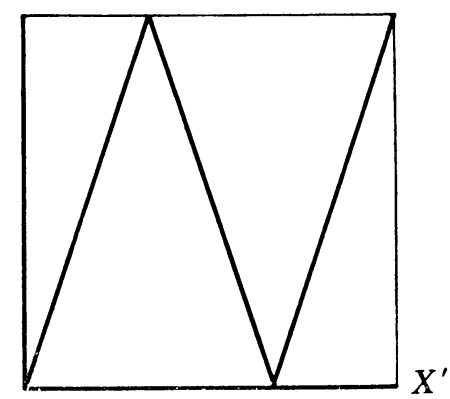

For $v>1$, we argue as follows. Let $\varepsilon<1$ and let $\mathfrak{A}_{\varepsilon}$ be the cover of $X^{\prime}$ consisting of all intervals of length $\leqq \varepsilon$ of the type $(a, b),,[0, b)$ or $(a, \pi]$. For such an interval $I$ of length $l, \psi^{-1} I$ is the union of disjoint similar intervals each of length $l^{\prime}$ where $l / v \leqq l^{\prime} \leqq 2 l / v$; this is clear from Figure 2. Hence $\psi^{-1} \mathfrak{A}_{\varepsilon} \prec \mathfrak{A}_{\varepsilon, v}$. By properties (1) and (4) of the introduction it follows that $\psi^{-k} \mathfrak{A}_{\varepsilon} \prec \mathfrak{A}_{\varepsilon / v^{k}}$ for $k=1,2$, Hence

$$
\mathfrak{A}_{\varepsilon} \vee \psi^{-1} \mathfrak{A}_{\varepsilon} \vee \cdots \vee \psi^{-n} \mathfrak{A}_{\varepsilon} \prec \mathfrak{A}_{\varepsilon} \vee \mathfrak{A}_{\varepsilon / v} \vee \cdots \vee \mathfrak{A}_{\varepsilon / v^{n}}=\mathfrak{A}_{\varepsilon / v^{n}},
$$

since $\mathfrak{A}_{\varepsilon / v} r \prec \mathfrak{A}_{\varepsilon / v} n$ for $0 \leqq r \leqq n$. Therefore, by property (3),

$$
N\left(\mathfrak{A}_{\varepsilon} \vee \psi^{-1} \mathfrak{A}_{\varepsilon} \vee \cdots \vee \psi^{-n} \mathfrak{A}_{\varepsilon}\right) \leqq N\left(\mathfrak{A}_{\varepsilon / v^{n}}\right) \leqq \pi v^{n} / \varepsilon+1 .
$$

Therefore $h\left(\psi, \mathfrak{A}_{\varepsilon}\right) \leqq \log v$. Now the sequence $\left\{\mathfrak{A}_{1 / n}\right\}$ is shown in [1] to be a refining sequence and so, by property $(6), h(\psi)=\lim _{n \rightarrow \infty} h\left(\psi, \mathfrak{A}_{1 / n}\right) \leqq \log v$. 
Next we will prove the reverse inequality, $h(\psi) \geqq \log v$. Let $\mu$ be Lebesgue measure on $X^{\prime}$ and let $g(\mathfrak{B}, x)$ be defined for $x \in X^{\prime}$ and $\mathfrak{B}$ a cover of $X^{\prime}$, as in Lemma 1. Suppose now that $\varepsilon<\pi / 2 v$. We note first that if $\mathfrak{B}$ is an open cover whose sets have diameter $<v \varepsilon$,

$$
g\left(\psi^{-} \mathfrak{B} \vee \mathfrak{A}_{\varepsilon}, x\right) \geqq g(\mathfrak{B}, \psi x),
$$

and, for $x \notin S_{\varepsilon}$,

$$
g\left(\psi^{-1} \mathfrak{B} \bigvee \mathfrak{U}_{\varepsilon}, x\right)=v g(\mathfrak{B}, \psi x),
$$

where $S_{\varepsilon}$ is the set of points at distance $\leqq \varepsilon$ from some $\pi k / v$ with $0<k<v$. The proof of (3.1) and (3.2) is immediate from Figure 2, where $B$ represents some set of $\mathfrak{B}$. Inequality (3.1) follows since $\mu\left(\psi^{-1} B\right)=\mu(B)$ and $\mu\left(\psi^{-1} B \cap A\right) \leqq \mu\left(\psi^{-1} B\right)$ for any $A$. For (3.2), the essential point is that for any $B \in \mathfrak{B}, \psi^{-1} B$ consists of exactly $v$ pieces of each measure $\mu(B) / v$ and di-

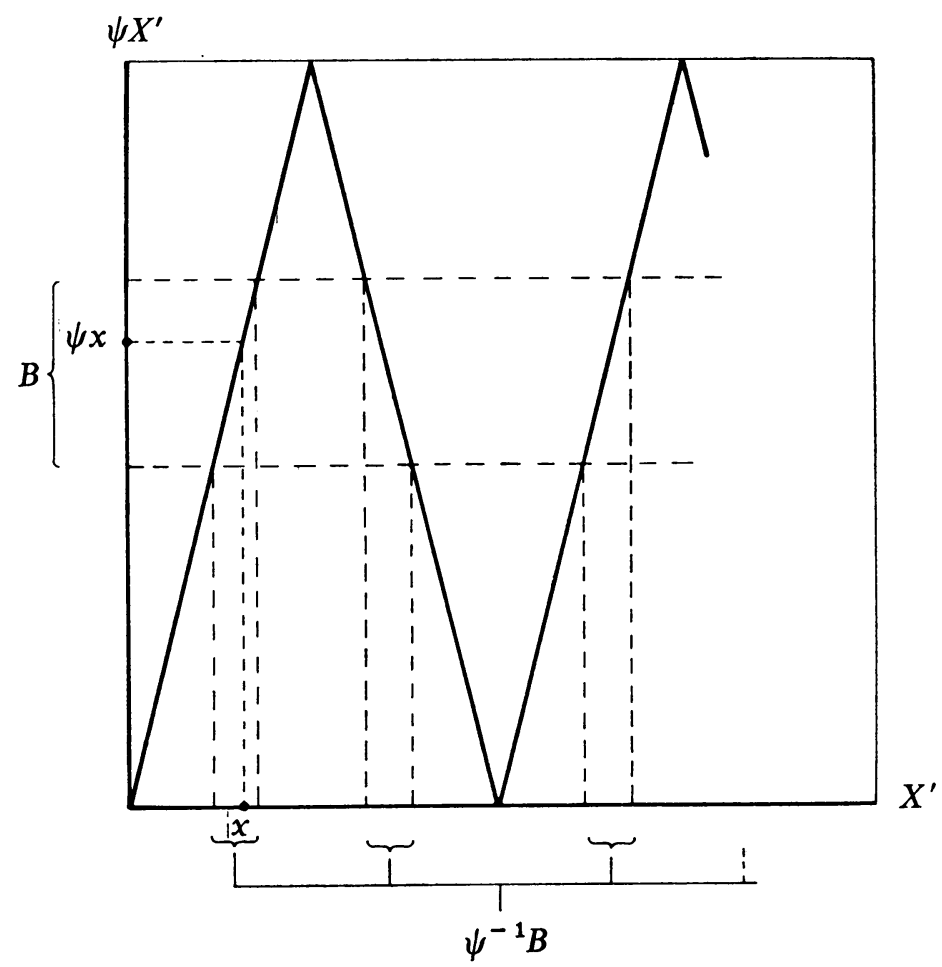

FIGURE 2

ameter $d(B) / v$ where $d(B)$ is the diameter of $B$. If $x \notin S_{\varepsilon}$ and $x \in A \in \mathfrak{A}_{\varepsilon}$ then $A \cap \psi^{-1} B$ contains points of at most one such piece and there is a choice of 
$A \in \mathfrak{A}_{\varepsilon}$ such that $A \cap \psi^{-1} B$ is the whole of one piece. Let $g_{n}(x)$ denote $g\left(\mathfrak{A}_{\varepsilon} \vee \psi^{-1} \mathfrak{A}_{\varepsilon} \vee \cdots \vee \psi^{-n} \mathfrak{A}_{\varepsilon}, x\right)$. Taking $\mathfrak{B}=\mathfrak{A}_{\varepsilon^{\prime}} \bigvee \psi^{-1} \mathfrak{A}_{\varepsilon} \vee \cdots \vee \psi^{-n} \mathfrak{A}_{\varepsilon}$ in (3.1) and (3.2), we obtain

$$
g_{n+1}(x) \geqq g_{n}(\psi x),
$$

and, for $x \notin S_{\varepsilon}$,

$$
g_{n+1}(x)=v g_{n}(\psi x) .
$$

From (3.3) and (3.4) we have, for $0 \leqq k \leqq v-1$,

$$
\begin{aligned}
\int_{k \pi / v}^{(k+1) \pi / v} g_{n+1}(x) d x & \geqq \int_{k \pi / v+\varepsilon}^{(k+1) \pi / v-\varepsilon} v g_{n}(\psi x) d x+\int_{k \pi / v}^{k \pi / v+\varepsilon} g_{n}(\psi x) d x+\int_{(k+1) \pi / v-\varepsilon}^{(k+1) \pi / v} g_{n}(\psi x) d x \\
& =\int_{v \varepsilon}^{\pi-v \varepsilon} g_{n}(y) d y+v^{-1} \int_{0}^{v \varepsilon} g_{n}(y) d y+v^{-1} \int_{\pi-v \varepsilon}^{\pi} g_{n}(y) d y .
\end{aligned}
$$

Hence

$$
\int_{0}^{\pi} g_{n+1}(x) d x \geqq v \int_{0}^{\pi} g_{n}(y) d y-(v-1) \int_{0}^{v \varepsilon} g_{n}(y) d y-(v-1) \int_{\pi-v \varepsilon}^{\pi} g_{n}(y) d y .
$$

Now for $0<a<\pi / v-\varepsilon,[0, a] \cap S_{\varepsilon}=\phi$. Hence for $n \geqq 1$,

$$
\int_{0}^{a} g_{n}(x) d x=\int_{0}^{a} v g_{n-1}(\psi x) d x=\int_{0}^{v a} g_{n-1}(y) d y .
$$

Iterating this operation we have that if

$$
0<v^{r-1} a<\pi / v-\varepsilon \text { and } n \geqq r \geqq 0,
$$

then

$$
\int_{0}^{a} g_{n}(x) d x=\int_{0}^{a v^{r}} g_{n-r}(y) d y .
$$

Similarly if $v$ is odd (so that $\psi(\pi)=\pi$ ) and $a, n, r$ satisfy (3.6) then

$$
\int_{\pi-a}^{\pi} g_{n}(x) d x=\int_{\pi-a v^{r}}^{\pi} g_{n-r}(x) d x .
$$

Further (3.8) also holds if $v$ is even and $a, n, r$ satisfy (3.6). In this case $\psi(x)$ is an even function of $x-\pi / 2$ and cover $\mathfrak{A}_{\varepsilon}$ is symmetric about $\pi / 2$; hence $g_{n}(x)$ is an even function of $x-\pi / 2$ and now the left-hand and right-hand sides or (3.7) and (3.8) are respectively equal.

Let $I_{n}=\int_{0}^{\pi} g_{n}(x) d x$ and choose $r_{0}=r_{0}(\varepsilon)$ such that $0<\varepsilon v^{r_{0}-1}<\pi / v-\varepsilon$ and $2 v^{r_{0}} \varepsilon<\pi / v$. Let $1 \leqq s \leqq r_{0}$ and $n \geqq s-1$. Then from (3.5), 


$$
\begin{aligned}
I_{n+1} & \geqq v I_{n}-(v-1) \quad \int_{0}^{v \varepsilon} g_{n}(x) d x-(v-1) \int_{\pi-v \varepsilon}^{\pi} g_{n}(x) d x, \\
& =v I_{n}-(v-1) \quad \int_{0}^{v s_{\varepsilon}} g_{n-s+1}(y) d y-(v-1) \int_{\pi-v s_{\varepsilon}}^{\pi} g_{n-s+1}(y) d y,
\end{aligned}
$$

from (3.7) and (3.8) with $a=v \varepsilon$ and $r=s-1$. By definition of $r_{0}, v^{s} \varepsilon<\pi-v^{s} \varepsilon$, and clearly $g_{n}(x)>0$ for all $0 \leqq x \leqq \pi$. Hence from (3.9),

$$
I_{n+1}>v I_{n}-(v-1) I_{n-s+1}
$$

for $1 \leqq s \leqq r_{0}$ and $n \geqq s-1$. Let $\lambda(r)$ be defined as in Lemma 2 . Then

$$
\begin{aligned}
h\left(\psi, \mathfrak{A}_{\varepsilon}\right) & =\log \left(\lim _{n \rightarrow \infty} N^{1 / n}\left(\mathfrak{A}_{\varepsilon} \vee \psi^{-1} \mathfrak{A}_{\varepsilon} \vee \cdots \vee \psi^{-n+1} \mathfrak{A}_{\varepsilon}\right)\right) \\
& \geqq \log \left(\liminf _{n \rightarrow \infty} I_{n-1}^{1 / n}\right), \text { by Lemma } 1, \\
& \geqq \log \lambda\left(r_{0}\right), \quad \text { by Lemma } 2 .
\end{aligned}
$$

Letting $\varepsilon \rightarrow 0$ we may choose $r_{0}(\varepsilon)$ so that $r_{0} \rightarrow \infty$ and hence $h(\psi) \geqq \sup _{\varepsilon} h\left(\psi, \mathfrak{A}_{\varepsilon}\right)$ $\geqq \log v$ since $\lim _{r \rightarrow \infty} \lambda\left(r_{0}\right)=v$. This concludes the proof of the theorem.

\section{REFERENCES}

1. R. L. Adler, A. G. Konheim and M. H. McAndrew, Topological entropy, Trans. Amer. Math. Soc. 114 (1965), 309-319.

INTERNATIONAL Business MACHINes CoR PORATION, YoRKTOWN HEIGHTS, NEW YORK 